\title{
Correlation between Internal Derangement of the Temporo-Mandibular Joint and Ipsi-Lateral Mediotrusive Molar Interferences-A Condylographic Study Using Virtual Articulation
}

\author{
Markus Greven ${ }^{1,2 *}$, Eva Piehslinger ${ }^{2}$, Thomas Haberl ${ }^{3}$, and Christian Betzl ${ }^{4}$ \\ ${ }^{1}$ Private Office, Bonn, Germany \\ ${ }^{2}$ University Dental School, Department of Prosthodontics, Medical University of Vienna, Austria \\ ${ }^{3}$ Vienna School of Interdisciplinary Dentistry, Austria \\ ${ }^{4}$ Private Office, Munich, Germany
}

*Corresponding author: Markus Greven, Private Office, Bonn, Germany, Tel: 49228 985900; E-mail: markusgreven@t-online.de

Received: 27 May, 2020 | Accepted: 09 Jun, 2020 | Published: 24 Jun, 2020

Citation: Greven G, Piehslinger E, Haberl T, Betzl C (2020) Correlation between Internal Derangement of the Temporo-Mandibular Joint and Ipsi-Lateral Mediotrusive Molar Interferences-A Condylographic Study Using Virtual Articulation. Int J Dent Oral Health 6(5): dx.doi. org/10.16966/2378-7090.328

Copyright: (c) 2020 Greven G, et al. This is an open-access article distributed under the terms of the Creative Commons Attribution License, which permits unrestricted use, distribution, and reproduction in any medium, provided the original author and source are credited.

\begin{abstract}
Objective: Aim of this pilot study was to test if molar interferences in symptomatic patients are suggesting altered condylographic tracings in terms of an internal TMJ structural disturbance, in particular an increased transversal joint mobility higher than $0.6 \mathrm{~mm}$ in guided mediotrusive movements of both joints.

Methods: Eighty-three symptomatic patients-suffering from signs and symptoms of a Temporo-Mandibular Disorder (TMD) from a private dental office in Munich were diagnosed with an occlusion-dictated TMD according to the clinical protocol of the Vienna School of Interdisciplinary Dentisty (VieSID). The Sagittal Condyle Inclination (SCI) was measured by condylographic examination with the Cadiax system and compared to the wear facet inclination of the mounted models by the Cadias 3D software.

Results: Statistically there was no correlation between the facet inclinations and transversal joint mobility. The null hypothesis was confirmed.

Conclusion: The authors conclude that symptomatic patients develop a muscular avoidance pattern towards the disturbing facets during mandibular dynamics with the result of a "joint protecting" reaction without ligamentous loosening.
\end{abstract}

Keywords: Temporomandibular dysfunction; Sagittal condyle inclination; Condylographic tracings; Occlusal interferences; Transversal joint mobility

\section{Introduction}

The temporomandibular joint is a very complex bicondylar diarthrosis and the central component of the temporomandibular complex. Mainly the medial and lateral pterygoids muscles are guiding the mandible along the joint surface. This muscular movement can be initially disturbed by an inadequate tooth structure which potentially causes a muscular avoidance pattern. According to Tanaka E, et al. [1] degenerative changes in the disc-condyle-capsule relationship can be leading to a variety of morphological and functional deformities. Morphological adaptations to compromised loading pattern of the masticatory structures lead over time to changes within the joints compartments [2].

Hyperbalancing contacts are characterized by a steeper inclination than the sagittal inclination of the glenoid fossa to Axis Orbital Plane
(AOP). Orthobalancing contact areas are flatter or have the same inclination as the eminentia articularis. The teeth surfaces are not guiding but they can control the movement in this case.

Though the anatomical joint space's dimensions are limited in medial, dorsal, cranial and lateral aspects the TMJ should show, induced by necessity, an asymetrically increased transversal mobility (Figures 1a and 1b). Christiansen G [3] is reporting of a supposedly pathological amount of lateral mobility in the lower compartment of more than $0.6-0.8 \mathrm{~mm}$.

Christiansen G [3] measured that condyles show limited capacity in medial direction associated with a retral and cranial sliding. He stated regular seated condyles allow manipulation in the green sectors in frontal and sagittal view: condyles in a functional joint space (Figure 2). 


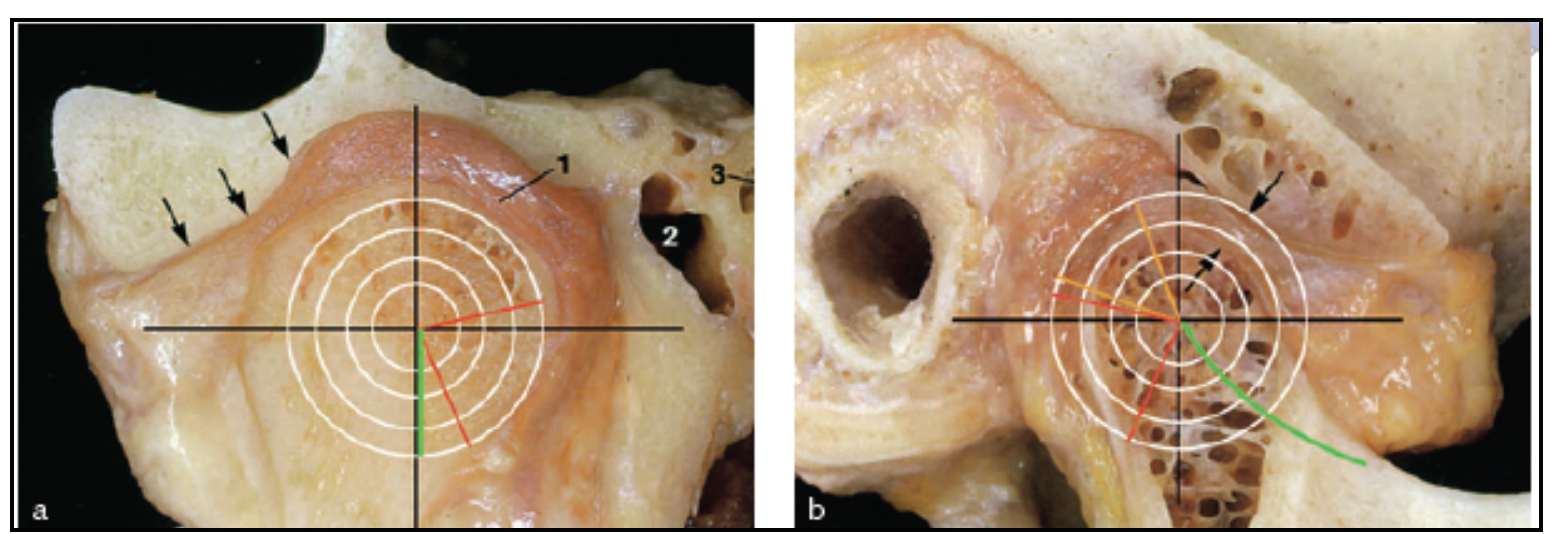

Figure 1: Healthy subject's motion capacity in transversal (a) and sagittal (b) direction. Starting position is ICP. Physiologically, during dynamics the condyle should glide along the pathways marked in green.

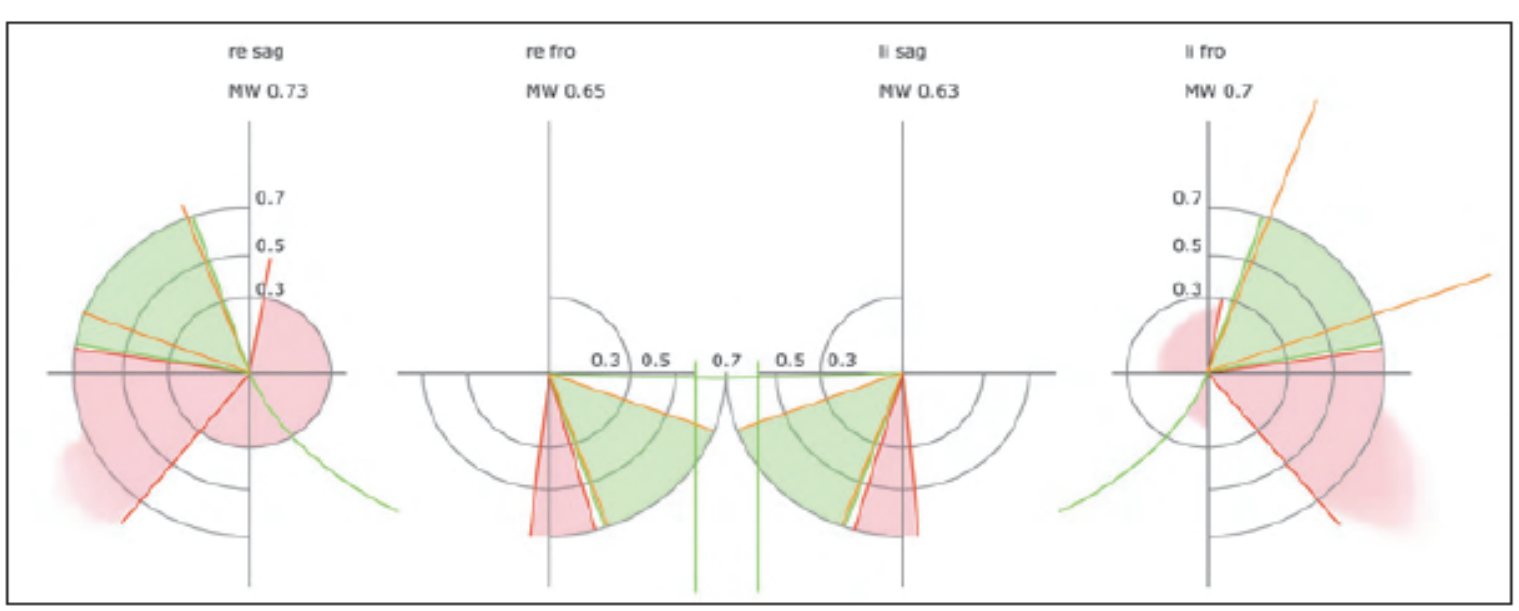

Figure 2: Condyles show a physiologic movement capacity in the green area. Condyles with dysfunctional trajectory deflect at least in one of the red zones.

Alterations to a normal translation and opening rotation can be detected by computerized condylography as a sign of an occlusiondictated avoidance pattern. Greven M, et al. [4] conclude from their fMRI study that brain activation and unpleasantness of the volunteers increases with a level of condylar displacement beyond $0.5 \mathrm{~mm}$ for the amygdalae ("flight-or-fight"-reaction) and $0.7 \mathrm{~mm}$ for the prefrontal area ("awareness" of a discomfortable situation) [5]. All structures and joint components are under loading condition when deviated and/or deflected by the amount of $0.6-0.8 \mathrm{~mm}$ in retrusive, surtrusive and/or transversal direction [6].

Farrar WB, et al. [7] defined internal derangement in several degrees of disc and condyle disunification. Wilkes classified the tissue damages of the TMJs from slight changes in the condyle and disc relationship to the last stage of arthrotic damages with osteophytes resorbing the condyle bony structure [2]. The authors looked for an increased transversal mobility as a precursor of a disunification of condyle, articular disc and glenoid fossa (stage one to two ("Ligamentous loosening"/"Subluxation" according to Wilkes classification).
Computerized condylographic tracings (Cadiax, Fa. Gamma, Klosterneuburg, Austria) were used to detect the condylar pathway in terms of increased transversal mobility between excursive and incursive movements.

According to several authors computerized condylography is described as a reliable and non invasive tool in diagnostics and also in the treatment of the temporomandibular system [8-10]. Compared to static MRI or CBCT images condylography shows additional information about structural changes of the fossa-disccondyle complex [11] especially in dynamic situations and functional movements.

\section{Objectives of the Study}

To identify molar wear facets as a predictor for a transversal pathway shift of the condyle. To quantify the inclination and length of mediotrusive wear facets as indicative.

If so, what kind of association can be found? Positive association is defined as following: the length and inclination increases, the 
transversal separation also increases. Negative association: if length and inclination increases, the separation decreases.

Try to define a threshold for the range of variability of inclination and length of wear facets that might suggest risk for TMD in terms of ligamentous loosening.

\section{Accordingly, the formulated null hypothesis for this study:}

- Inclination and length of mediotrusive wear facets do not predict increased transversal tracing separation in condylographic tracings.

\section{Materials and Methods}

A pilot condylographic study was done with 83 symptomatic patients (22 male; 61 female), asking for a functional diagnostic treatment with a splint between 2014 and 2015 in a private dental clinic in Munich.

\section{The inclusion criteria were}

- At least 3 positive signs $(+,++)$ at the NMS palpation and/or positive signs $(+,++)$ at the CMS palpation or joint's capsule

- $\quad$ Evidence of MLT during guided asymmetric movements in the 3D condylographic registration

- Dentulous patients

- Partial edentulous patients with implant supported fixed prosthesis

- $\quad$ Abraded molar tooth surface with at least $2 \mathrm{~mm}$ of length

- $\quad$ A given consent

\section{The technical equipment used was}

- Condylograph and Cadiax III Diagnostic (Fa. Gamma, Klosterneuburg, Austria)

- Reference SL Articulator (Fa. Gamma, Klosterneuburg, Austria)

- Smartoptics 855 scanner (Fa. Gamma, Klosterneuburg, Austria)
- Gamma 3D dental software (Fa. Gamma, Klosterneuburg, Austria)

All patients took part in a standardized process according to the clinical protocol of VieSID: special medical anamnesis, dental history and occlusal index, neuromuscular and temporomandibular systems palpation, preliminary brainstem nerve analysis, radiographs, occlusiograms, waxplate RP recordings, casts analysis and condylography with the Cadiax system were done to diagnose an occlusion-dictated TMD.

The parameters examined were facet length (in millimeters) and facet inclination (in degrees) as well as the transversal joint play ("separation" of excursive and incursive movements) between the disc and condylar union, SCI and the amount of opening rotational movement.

Mediotrusive movements starting in Reference Position (RP) and under manual guidance of the examiner were analyzed according to the occurrence of a mandibular latero-deviation during incursive and excursive movements. The maximum transversal tracing separation value happening in each joint within the first $3 \mathrm{~mm}$ in between startand endpoint of the mediotrusive movement was considered. The upper jaw models were mounted to the AOP coordinate system, the lower jaw models were mounted with a RP-bite registration plate.

To identify the facets on the plaster models four steep and abraded molar surfaces were marked with a pencil and measured by a Korkhaus compass (Figure 3). Facets with a length of at least $2 \mathrm{~mm}$ in straight areas situated close to the maximal intercuspation were chosen.

To scan the mounted models according to AOP, Smartoptics 855 scanner with the Gamma Reference adapter was used. The Cadias 3D software imported the condylographic data from each patient and the RP mounted model data. The casts were analysed by using the virtual articulation tool of the Cadias 3D software (Figure 4).

For each patient the inclination and length of four wear facets were measured. If all four facets were equal or flatter than the contralateral joint's SCI the joint data were conjugated into the orthobalance group. If one or more facets were steeper than the contralateral SCI, the joint data were calculated within the hyperbalance group. This pilot study

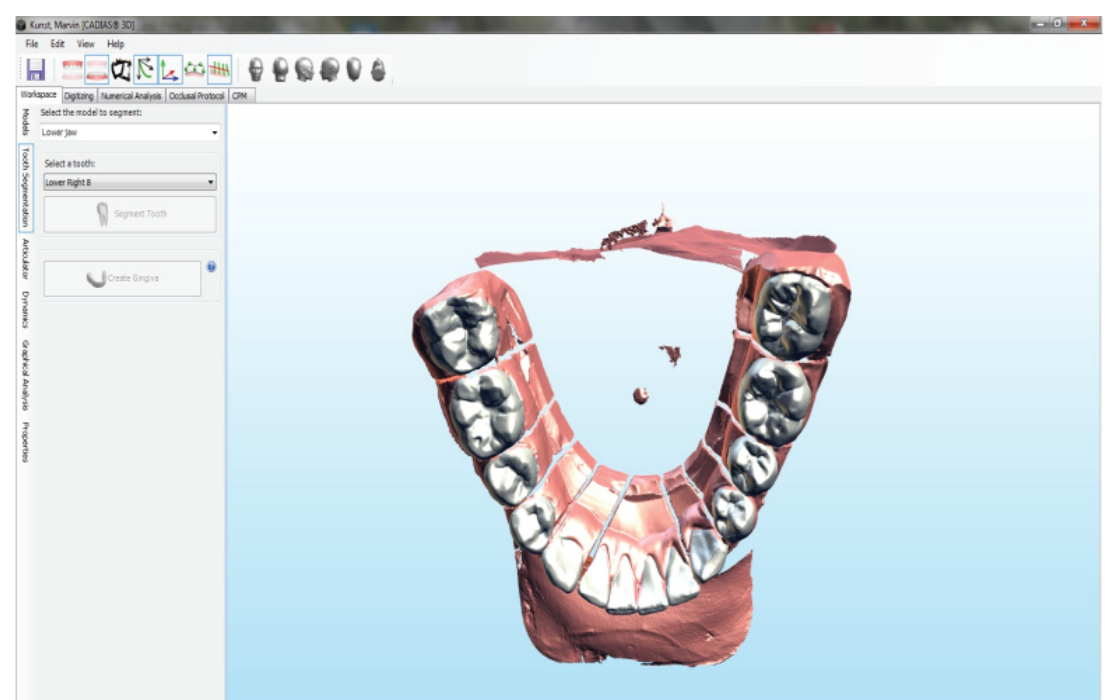

Figure 3: Marked plaster model surfaces showing facets Korkhaus compass. 


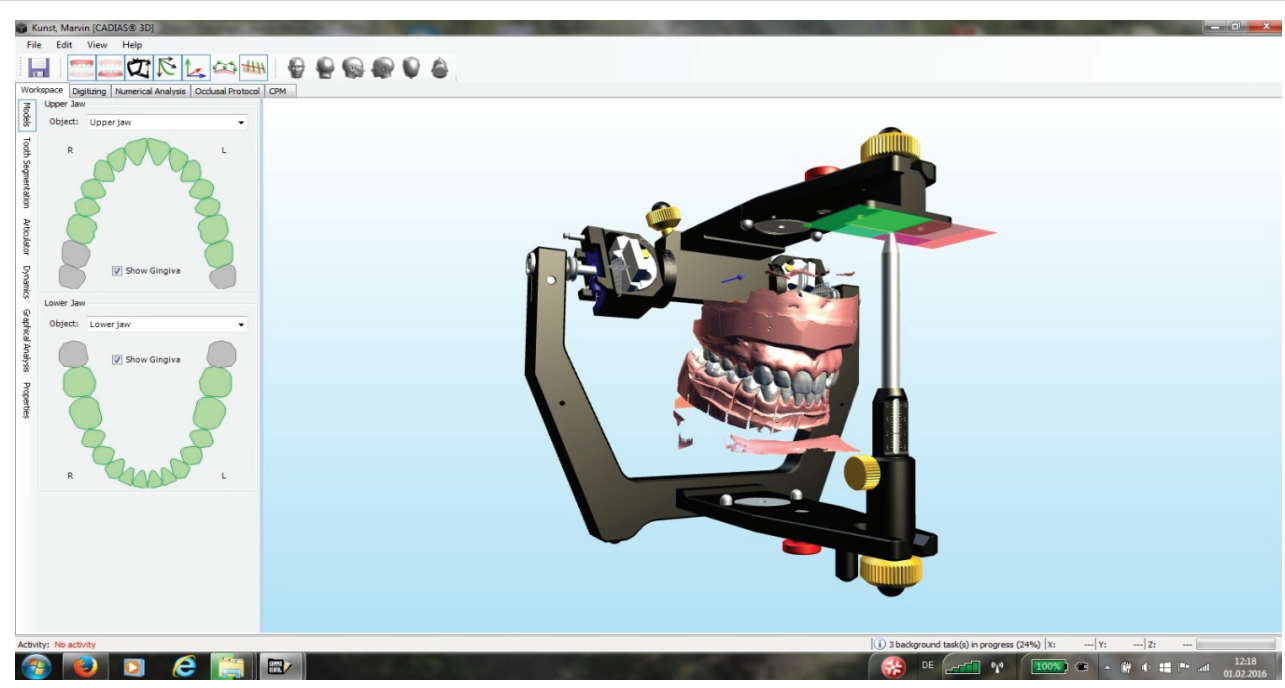

Figure 4: The digital models in the virtual articulator.

is using the calculated facet length feature of the Cadias 3D software.

With this investigation, one cause of TMJ problems that can contribute to a cranio-mandibular-dysfunction was tested: findings in the patient's occlusion.

\section{Results}

Statistically there was no correlation between the facet inclinations and transversal joint mobility. The quick and easy accessible intraoral finding of wear facets is neither predicting a positive nor a negative correlation to the joints transversal mobility. The null hypothesis was confirmed, the first objective has to be rejected upon the findings.

Ninety-four point nine $\%$ of all facets are orthobalacing contact areas and not steeper than SCI angulation to AOP. In 13 out of 83 symptomatic patients 17 out of 332 facets are steeper inclined than the corresponding joint surfaces. Five out of 17 hyperbalancing facets are shorter than $3 \mathrm{~mm}$ in length (Table 1). Neither the inclination nor the facet length is a reliable clinical predictor for ligamentous loosening in the lower compartment of the TMJ, just looking at mediotrusive guided movements only. Thirteen symptomatic patients are having hyperbalancing molar contacts but are showing as an average value less than $0.5 \mathrm{~mm}$ transversal separation of the mediotrusive tracks. Only 3 patients are having 3 hyperbalancing facets and showing an increased transversal mobility over $0.5 \mathrm{~mm}$.

Three out of 13 patients are having hyperbalancing facets in both joints. Two out 13 patients are showing more than $0.5 \mathrm{~mm}$ of tracing separation (Table 2). One patient is showing increased transversal mobility only in one joint.

In the hyperbalance group the mean facet inclination increases, while the mean value for the facet length stays more or less the same. Regarding the negative association of the second objective the transversal capacity has a tendency to decrease, while the facet inclination's mean value increases and the facet length stays the same. More opening rotation might suggest one of the masticatory organ's ways to hurdle the hyperbalances. But the analyzed joints and casts data are not indicating a direction or treshold for the range of variability that might suggest a risk for ligamentous loosening in the lower compartment of the TMJs.

\section{Conclusion}

This pilot study was done in order to test if molar wear facets are a good clinical predictor for ligamentous loosening in the TMJ. As it is described in the literature loose ligaments are early signs of a structural integrity loss $[12,13]$. Any correlation to a patient's joint status would be of clinical favor to obtain, before doing irreversible dental treatments. These facets are easy to detect, and so valid information about a patient's clinical risk of having a compromised status in the TMJ could be find. Consciousness about the joint's condition should influence clinical decision making. Christiansen G. [14] stated that our success or failure in prosthodontics is closely related to an appropriate management of mediotrusive movements. If a dental treatment is getting irreversible, the importance of staging the patient's joint condition priorly is very high in order to provide a successful and long lasting treatment.

For more than a century several ideas were provided how teeth have to occlude and disocclude and how missing teeth should be reconstructed in order to serve without interfering.

The occlusal concept of sequential occlusion with canine dominance was created by Slavicek, Gausch and Kulmer in the early 1980s. According to Prof. Slavicek R [15] functional dividing plane hypothesis the occlusal task for these two pairs of molars is only to support the transmission of occlusal forces in the palatal arch in a static way, without interfering in dynamics. This concept is helping with well defined leeway spaces to prevent posterior interferences and allow at the same time to reconstruct occlusal surfaces which are providing a maximum of chewing efficiency.

The authors are suggesting seeing facets on molars as a clinical risk factor, not only in terms of early material fatigue, but also in keeping and maintaining the patient's masticatory organ in healthy conditions throughout a prosthodontic lifespan of a restauration.

Condylography is the tool, despite an eye opening tool for a proper diagnosis of the whole masticatory organ, to obtain a clear three dimensional reconstruction plan for prosthodontics and orthodontics. Therefore it is possible in any treatment to control the occlusal forces by its increased precision in articulator settings to reduce the body's necessity in adaptation [16]. 
Table 1: Distribution of facet inclination and facet length, $\mathrm{SCl}$.

\begin{tabular}{|l|c|c|c|}
\hline \multicolumn{1}{|c|}{ Facets summery } & $\mathbf{1 6 6}$ Joints total $\mathbf{3 3 2}$ facets & 26 Joints having $\mathbf{1 7}$ hyper/35 ortho & $\mathbf{1 4 0}$ Joints $\mathbf{2 8 0}$ orthobalances \\
\hline Facet inclination upper jaw & $29.53^{\circ}\left(1.52^{\circ} / 59.51^{\circ}\right)$ & $33.17^{\circ}\left(7.1^{\circ} / 59.51^{\circ}\right)$ & $28.86^{\circ}\left(1,52^{\circ} / 56,82^{\circ}\right)$ \\
\hline Facet inclination lower jaw & $29.61^{\circ}\left(1.21^{\circ} / 70.05^{\circ}\right)$ & $33.60^{\circ}\left(3.39^{\circ} / 70.05^{\circ}\right)$ & $28.88^{\circ}\left(1.21^{\circ} / 54.7^{\circ}\right)$ \\
\hline SCl right & $51.37^{\circ}\left(31.33^{\circ} / 82.17^{\circ}\right)$ & $41.48^{\circ}\left(31.33^{\circ} / 53.37^{\circ}\right)$ & $53.21^{\circ}\left(33.1^{\circ} / 82.17^{\circ}\right)$ \\
\hline SCI left & $54.01^{\circ}\left(30,59^{\circ} / 64.44^{\circ}\right)$ & $45.01^{\circ}\left(30.59^{\circ} / 54.2^{\circ}\right)$ & $55.29^{\circ}\left(36.68^{\circ} / 69.54^{\circ}\right)$ \\
\hline Facet length upper jaw & $3.5 \mathrm{~mm}(2.2 \mathrm{~mm} / 4.9 \mathrm{~mm})$ & $3.4 \mathrm{~mm}(2.2 \mathrm{~mm} / 4.9 \mathrm{~mm})$ & $3.5 \mathrm{~mm}(2.2 \mathrm{~mm} / 4.6 \mathrm{~mm})$ \\
\hline Facet length lower jaw & $3.4 \mathrm{~mm}(1.7 \mathrm{~mm} / 5.0 \mathrm{~mm})$ & $3.4 \mathrm{~mm}(4.8 \mathrm{~mm} / 4.2 \mathrm{~mm})$ & $3.4 \mathrm{~mm}(1.7 \mathrm{~mm} / 5.0 \mathrm{~mm})$ \\
\hline
\end{tabular}

Table 2: Distribution of open rotation and transversal joint mobility.

\begin{tabular}{|l|c|c|c|}
\hline \multicolumn{1}{|c|}{ Joint parameters } & $\begin{array}{c}\text { Total 166 Joints (83 symp. Pat. } 332 \\
\text { Facets) }\end{array}$ & $\begin{array}{c}\text { 26 Joints (13 symp. Pat.) } \\
\text { 17 hyper } / 35 \text { ortho }\end{array}$ & $\begin{array}{c}\text { 140 Joints (70 symp. Pat.) } \\
\text { 280 orthobalances }\end{array}$ \\
\hline Gamma right & $2.84^{\circ}\left(0.4^{\circ} / 11.3^{\circ}\right)$ & $3.19^{\circ}\left(1.53^{\circ} / 6.29^{\circ}\right)$ & $2.77^{\circ}\left(0.4^{\circ} / 11.03^{\circ}\right)$ \\
\hline Gamma left & $2.33^{\circ}\left(0.36^{\circ} / 6.41^{\circ}\right)$ & $33.60^{\circ}\left(0.94^{\circ} / 5.77^{\circ}\right)$ & $28.88^{\circ}\left(0.36^{\circ} / 6.14^{\circ}\right)$ \\
\hline Delta y-MR & $0.46 \mathrm{~mm}(0.0 \mathrm{~mm} / 1.8 \mathrm{~mm})$ & $0.29 \mathrm{~mm}(0.09 \mathrm{~mm} / 0.68 \mathrm{~mm})$ & $0.49 \mathrm{~mm}(0.0 \mathrm{~mm} / 1.8 \mathrm{~mm})$ \\
\hline Delta y-ML & $0.52 \mathrm{~mm}(0.02 \mathrm{~mm} / 2.16 \mathrm{~mm})$ & $0.39(0.08 \mathrm{~mm} / 1.19 \mathrm{~mm})$ & $0.54 \mathrm{~mm}(0.02 \mathrm{~mm} / 2.16 \mathrm{~mm})$ \\
\hline
\end{tabular}

Tamaki K, et al [17] observed a muscular avoidance pattern and an increased opening rotation of the mandible by using electromyography and computed condylography in his studies.

One important supposition is that symptomatic patients try to avoid their disturbing facets in dynamics. By combining several avoidance patterns, the TMJ could react up to a certain threshold self defending and joint protecting without ligamentous loosening. Nevertheless it is of very high importance to know and implement the patient's individual joint parameters for joints diagnosed prior to irreversible treatment.

Obtaining the results of the present study, it is planned to prove in the future after additional research, that interferences on molars are compromising the joints integrity in terms of undisturbed function. In a pilot subject out of this cohort areas of tooth contacts were found in the functional movement's bruxing, chewing and deglutition, explaining the creation of long abraded surfaces by the opponent teeth.

The simulation of all kind of recorded movements in the virtual environment of Cadias 3D can be verified in the patient with bruxchecker detection foils, concerning nighttime bruxism and deglutination. This may help to clarify the objectives tested in this pilot study.

\section{Acknowledgment}

First of all I want to thank my family and colleagues, who were always appreciative, full of help and supportive criticism to my work.

Thank you, also to Thomas Haberl for helping me with a customisation of my Cadias 3D software, which allowed me to measure facet inclinations.

My huge appreciation goes also to Dr. Cinzia Fornai for explaining me the way to ask questions, which can or cannot be answered in an academic way.

Last but not least, i would like thank my postgraduate teachers in Vienna and Japan.

\section{Declaration of Interest Statement}

The authors report no conflict of interest.

\section{References}

1. Tanaka E, Detamore MS, Mercuri LG (2008) Degenerative Disorders of the Temporomandibular Joint: Etiology, Diagnosis, and Treatment. J Dent Res 87: 296-307.

2. Wilkes $\mathrm{CH}$ (1989) Internal Derangements of the Temporomandibular Joint. Pathological Variations. Arch Otolaryngol Head Neck Surg 115: 469-477.

3. Christiansen G (2010) Computer-aided measurement of the functional joint space of the temporomandibular joint. J Craniomandibular Function 2: 329-343.

4. Greven M, Otsuka T, Zutz L, Weber B, Elger C, et al. (2011) The Amount of TMJ Displacement Correlates With Brain Activity. Cranio 29: 291-296.

5. Otsuka T, Watanabe K, Hirano Y, Kubo K, Miyake S, et al. (2009) Effects of mandibular deviation on brain activation during clenching: an fMRI preliminary study. Cranio 27: 88-93.

6. Greven M, Landry A, Carmignani A (2016) Comprehensive dental diagnosis and treatment planning for occlusal rehabilitation: a perspective. Cranio 34: 215-217.

7. Farrar WB, McCarty WL Jr (1979) Inferior joint space arthrography and characteristics of condylar paths in internal derangements of the TMJ. J Prosthet Dent 41: 548-555.

8. Ahlers MO, Bernhardt O, Jakstat HA, Kordaß B, Türp JC, et al. (2015) Motion analysis of the mandible : concept for standardized evaluation of computer-assisted recording of condylar movements. J Craniomandibular Function 6: 333-352.

9. Bonilla-Aragon $\mathrm{H}$, Tallents $\mathrm{RH}$, Katzberg RW, Kyrkanides $\mathrm{S}$, Moss ME (1999) Condyle position as a predictor of temporomandibular joint internal derangement. J Prosthet Dent 82: 205-208.

10. Piehslinger E, ErtI L (1995) Computerized axiography for standardized evaluation of TMJ function and dysfunction. Medinfo 8: 1303-1304.

11. Park B-K, Tokiwa O, Takezawa Y, Takahashi Y, Sasaguri K, et al. (2008) Relationship of tooth grinding pattern during sleep bruxism and temporomandibular joint status. Cranio 26: 8-15.

12. Crawford SD (1999) Condylar axis position, as determined by the occlusion and measured by the CPI instrument, and signs and symptoms of temporomandibular dysfunction. Angle Orthod 69: 103-115-116. 
13. Schellhas KL (1989) Internal Derangement of the Temporomandibular Joint: Radiologic Staging with Clinical, Surgical, and Pathologic Correlation. Magnetic Resonance Imaging 7: 495-515.

14. Christiansen G (2012) Das Kiefergelenk verstehen. Ingolstadt: CMD Compact KG.

15. Slavicek R (1984) Die funktionellen Determinanten des Kauorgans. University of Michigan.

16. Hellmann D, Schindler HJ, Schindler H (2015) Value of axiography in the reconstruction of occlusal function. Journal of Craniomandibular Function 7: 39-45.

17. Tamaki K, Hori N, Fujiwara M, Yoshino T, Toyoda M, et al. (2001) A pilot study on masticatory muscles activities during grinding movements in occlusion with different guiding areas on working side. Bull Kanagawa Dent Coll 29: 26-27. 